\title{
Keyword index to Volume 81
}

Acetaminophen 542

$\mathrm{N}$-Acetyltransferase 1537

$\mathrm{N}$-Acetyltransferase 2537

Actin 393

Acute lymphoblastic leukaemia 175

Acute myeloid leukaemia 900

Adenocarcinoma 159

Adenoma 232

Adenovirus vectors 277

Adolescence 167

Adrenal cortex 684

Adrenocortical tumours 300

Advanced non-small-cell lung cancer 846

Adverse effects 336

Aetiology 898, 907

Affective disorders 907

Age-period-cohort analysis 152

Age-period-cohort models 159

Agreements 87

ALA cream 13

ALA lotion 13

ALA-PDT 13

Alcohol abuse 907

Alkaline sphingomyelinase 232

Allelic imbalance 1111

5-Aminolaevulinic acid 13

Amplification 1174, 1410

Analgesics 542

Anatomic sites 528

Androgens 28

Androgens, synthesis of 622

Androgen ablation 242

Androgen receptors 242, 672

Angiogenesis 54, 225, 354, 647, 1088, 1335, 1363,1398

Angiosarcomas 532

Angiostatin 1269

Animal tumour models 638

Anthracyclines 24

Anthropometry 1257

Anti-angiogenesis 1269

Anti-vascular shutdown 1318

Antibodies 769

Antimicrobial peptides 393

Antisense interleukin-2 oligonucleotides 822

Antisense-EGFR 994

AP-2 133

APC protein 850

Apoptosis 277, 294, 387, 423, 520, 592, 747,

822, 952, 981, 1274, 1285, 1294

Arsenic trioxide 796

Asia, south east 893

Aspirin 294, 542

Astrocytomas 835

Atomic bomb survivors 1248

ATPase 269

Autoantibodies 702

Autocrine regulation 1335

Automatic quantification 1363

Azolyl steroids 622
Background transcription 870

BAG-1 1042

bcl-2 252

Bcl-2 387, 860, 1042

Bcl-x(L) 387

Benzamides 981

Berberine 416

Bi-specific monoclonal antibodies 431

Bicalutamide 242

Biological factors 925

Biomarkers 1222, 1238

Bioreductive drugs 1127

Biotinylated anti-CD3 Fab fragments 431

Bisdioxopiperazine 800

BK virus 898

Bladder cancer 537, 832

B-lymphoma cells, human 43

Body mass indices 890

Bombesin receptors 966

Bone marrow 1213

Brain tumours 1371

BRCAl 179

BRCA1 503

BRCA2 179

BRCA2 503

Breast cancer 225, 287, 316, 342, 387, 440, 532, 592, 690, 702, 850, 912, 1002, 1042, 1142, 1248, 1304, 1385

Breast cancer cells 252

Breast neoplasms 167, 490

Breast size 1257

Bromodeoxyuridine 404

Buthionine sulphoximine 796

C1311 367

CA19-9 769

CA 125662

Cachexia 80

Calcium 219

cAMP analogues 1134

Cancer chemotherapy 69

Cancer invasion 721

Cancer risks 672

Cancer screening 554

Cancer testis antigens 1080

Cancer therapy 966

Cancer xenografts, human 203

Carboplatin 336

Carcinomas 592, 1059, 1363

Carcinomas, human 822

Cardiotoxicity 24

Carotenoids 1234

Case-control studies $62,559,890$

Caspases 294, 592

$\beta$-Catenin 1392

Cathepsin-B 510

Cathepsin-D 1385

CD34 808

Cell adhesion 381
Cell cultures 814

Cell cycles 808, 1285

Cell cycle checkpoints 959

Cell cycle regulators 696

Cell lines 814

Cell proliferation 712

Cell transformation 790

Cell-cell interaction 1142

c-erbB-2 1385

c-erbB-2 oncogenes 790

Cervical cancer 95, 108, 159, 554, 989

Cervical dysplasia 1234

Cervical scrapings 114

CGH 300, 1328, 1410

CHART 1196

Chemotherapy 75, 316, 336, 469, 484, 520, $835,860,1022,1031,1213,1280$

Chemotherapy, high-dose sequential 449

Chemotherapy responses 1017

Childhood 900

Childhood cancer 549

Childhood leukaemia 144

Children 175, 336, 835

Chromogranin A 667

Chromosomal aberrations 1328

Chromosome 3684

Chromosome 9684

Chromosome 17q 1402

Chromosome 221150

Chromosome X 684

Cigarette smoking 537

Cimetidine 1356

Circulating cancer cells 1066

Circulating epithelial cells 832

Circulating tumour cells 1165

Cisplatin 28, 95, 469, 1294

Clinical outcomes 696, 1174

Clinical trials 1356

Clonogenicity 756

c-MET 43

c-myc 1385

Cofluent fibroblast layers 934

Cohort effects 152

Collagen 654

Collagen gene expression 1142

Colon adenocarcinoma cell lines 1285

Colon cancer 232, 237, 294, 600, 1274

Colon tumours 1318

Colorectal cancer 62, 122, 133, 190, 287,

$305,367,874,1009,1116$

Colorectal cancer, human 496

Combination regimens 609

Combined modality 1206

Combretastatin A-4 1318

Concurrent chemoradiation in NSCLC 310

Core biopsy 1088

Cost-effectiveness analysis 1243

COX-2 1274

CPT-11 95, 440

CT antigens 1162 
CTLs 342,881

Cultures 1344

Cyclic A 1017

Cyclic D1 127

Cyclin D 705

Cyclin D1 1174

Cyclin-dependent kinase 705

CYP17 polymorphisms 141

Cystoprostatectomy 832

Cytochrome P450 reductase 1127

Cytogenetics 6

Cytokeratin 8769

Cytokeratin 20870

Cytokines 359

Cytokine-induced killer cells 1009

Cytological screening 159

Cytoplasm 1174

Cytoreduction 733

Cytotoxicity 367, 1022, 1127

DCIS 1410

Dendritic cells 1280

Dialysis in vitro 330

Diet 1234,1238

Differentiation markers 855

Directly streptavidinylated antibodies 431

Dissemination 43

Distinct entities 586

Divalent Fab' 972

DNA damage 1127

DNA intercalation 367

DNA mutations 409

DNA repair 800

Docetaxel 457, 609

Docosahexaenoic acid 80,1238

Dose escalation 1022

Dosimetry 972

Doxorubicin 966

Drug binding 783

Drug delivery systems 1155

Drug targeting 404

Ductal carcinoma in situ 702

Early relapse 1385

E-cadherin 1103, 1392

ED-B fibronectin hybridization in situ 1071

EGF 1134

Eicosapentaenoic acid 80, 1238

Electromagnetic fields 377

ELISA 490

Endotoxins 1311

Ependymoma 1150

Epidemiology 62, 144, 672, 900

Epstein-Barr virus 1182

Establishment 814

Esthesioneuroblastoma 586

Evaluations 912

Experimental metastasis 756

External ear 528

Extracellular matrix 1142

Extracellular signal regulated kinase 1116

Extraction 600

False-negative tests 305

Familial clustering 1150

FAP 232

$\alpha$-Fetoprotein 1188

Fibronectin isoforms 1071

FISH 1165,1328

Fish oil 80,440
Flavopiridol 269

Fluorescence 600

Fluorouracil derivatives 484

Free fraction 330

G1 checkpoints 696

Gastric cancer 19, 484, 647, 1356, 1392

G-CSF 1031

Gemcitabine 609, 846

Gene transfer 1009, 1122

Gene-specific repairs 1294

Genetics 672

Genetic markers 1002

Genetic susceptibility 179,1262

Geographic variations 1262

Germlines 409

GF120918 942

Glioblastoma 994

Glioma 835, 1371

Glucose 1,6-bisphosphate 219

Glucosylceramide 423

Glutathione 75, 796, 989

Glycolysis 219

Glycosylation 1188

Granzyme B 881

Growth inhibition 952

Haemangiosarcomas 532

Haematopoiesis 808

Haemoccult II ${ }^{\mathrm{R}} 305$

Haemophilus influenzae 175

H2-Antagonists 1356

HBsAg carriers 69

HBV profiles 69

Head and neck cancer 323, 457, 1196

Head and neck tumours 677

Hearts, chicken 934

Hepatocellular carcinoma 393, 747, 814, 1188

Hepatocyte growth factor 721

HER2/neu 1419

Hexokinase 219

HGF 194

HGF/SF 43

hGRPR 966

HHV-8 893

High-dose sequential chemotherapy 449

Histological grades 167

Histology 152

HIT genes 874

HLA 342

hNMBR 966

Hodgkin's disease 476, 1182

Hormone analogues 966

HPV 114

HT-29 294

HT-29 cells 367

Human topoisomerase I 800

Human cultured dendritic cells 1280

Human herpesvirus-8 893

Humanized monoclonal antibodies 1419

Hybrid capture 554

Hybridization in situ 496, 712

$17 \alpha$-Hydroxylase $/ \mathrm{C}_{1720}$-lyase inhibitors 622

Hypermethylation 677

Hypoxia 989, 1127

ICE 277,1031

I1307K 850

Image analysis 1363
Image cytometry 989

Imbalances 684

Immortalization 377

Immunoassays 122

Immunoconjugates 1155

Immunocytochemistry 404, 1213

Immunohistochemistry $127,747,1080$

Immunotherapy 1080,1280

In situ hybridization 496, 712

In vitro dialysis 330

Induction chemotherapy 310

Infection 549

Inflammatory breast cancer 449

Inoperability 1031

Interleukin-1 $\beta 277$

Interleukin-1ra 277

Interleukin-2 822, 1009

Interleukin-2/interleukin-2R pathways 822

Interleukin-6 690

Interleukin-8 647

International studies 463

Interphase cytogenetics 684

Interstitial photodynamic therapies 631

Interval cancer 912

Intestinal side-effects 440

Intraluminal seeding 6

Intramedullary tumours 835

Invasion 654,774

Ionizing radiation 959

Irinotecan 440

(Iso)flavonoids 269

Japan 1248

JC virus 898

JM216 1294

Kaposi's sarcoma associated herpesvirus 893

$\mathrm{K}^{+}$-ATPase 28

Ki-67 747, 1017

Kinase activities 705

KN62 959

Knock out mice 359

KSHV 893

Laminin isoforms 1071

Leukaemia 549, 833, 898, 1162, 1398

Leukaemia, acute lymphoblastic 175

Leukaemia, acute myeloid 900

Lipopolysaccharides 1311

Liver metastasis 600

LNCaP cells 242, 622

Lobular breast cancer 1103

Locally advanced cancer 1031

Locoregional recurrence 1385

LOH 108, 503, 677, 1103, 1111, 1371, 1410

Loss of heterozygosity $108,503,677,1103$,

$1111,1371,1410$

Luminometric immunoassays 122

Lung cancer 705, 1127, 1243

Lung surgery 721

Lymphangiogenesis 54

Lymphangiosarcomas 532

Lymphocyte function 1182

Lymphoedemas 532

Lymphomas 1009

B-Lymphoma cells, human 43

Lysosomes 367

Macrophages 37, 496

MACS 1165 
Magnetic resonance imaging 616, 638

Male breast cancer 141

Malignant mesothelioma 54, 1111, 1344

Mammalian cells 377

Mammary cancer 409,1335

Mammary tumours 194

Mammographic parenchymal patterns 1257

MAPK 1134

Marimastat 19

Markers 741

Mastectomy 1222

Matrigel 934

Matrix metalloproteinases (MMPs) 19, 287, 774

MCP-1 855

MEK 1116

Melanomas 219, 774, 918, 1066

Meningioma 381

Menopause 225

MES-Dx 5 cells 942

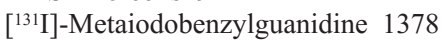

Metallothionein 712

Metastasis $287,316,712,774,814,832$, 1274,1311

Metastasis-associated factors 814

Metastatic potential 756

Methotrexate 316, 1037

MHC class I 881

MHC class II 808

MIA 1066

$\beta_{2}$-Microglobulin 881

Micrometastases 870, 1002, 1213

Microsatellites 1371

Microsatellite instability 108,190

Microsatellite marker analysis 1111

Microvessels 484

Microvessel count 1088

Microvessel density 1363

Minimally invasive debulking 631

Mismatched DNA 212

Mitoxantrone 316

MMP 1274

MN/CA IX/G250 antigens 741

$M N / C A 9 / G 250$ genes 741

Models 1243

Molecular analysis 586

Molecular cytogenetics 1328

Monoclonal antibodies 1155

Monoclonality 6

MRP1 269

MSI 108,190

mTHPC 37

MTS 1280

MUC1 mucin 431

Multidrug resistance $269,423,783,942$, 1304

Multifocal uroepithelial carcinomas 6

Multiple myeloma 952

Murine tumours 1311

Mutations 237, 1103

Myelodysplasia 1398

Myeloma 1162

Myofibroblasts 1071

NADPH 1127

Nasopharyngeal carcinoma 1122

Neoadjuvant chemotherapy 95, 1206

Neoplasms 684

Neoplasms, breast 907

Neoplasm staging 167
Nephrotoxicity 336

Neuroblastoma 1378, 1402

Neuroendocrine carcinoma 1351

Neurotic disorders 907

NFкB 981

Nipple aspirate fluid 1222

Nitric oxide 37

NK cells 881

nm23 469

Node-negative breast carcinoma 727

Non-Hodgkin's lymphoma 144, 860

Non-seminomatous germ cell tumours 1188

Non-small-cell lung cancer 127, 510, 769, 1031

Non-small-cell lung cancer, advanced 846

Non-small-cell lung cancer cell lines 609

Non-small-cell lung cancer primary cultures 609

Non-specified abuse 907

Non-steroidal anti-inflammatory drugs 62 , 542

NSAIDs 62,542

Nucleus 1174

Nude rats 1344

Nutritional supplementation 80

Obesity 890

Oesophageal cancer 712

Oesophageal squamous cell carcinoma 469

17ß-Oestradiol 387

Oestrogen 918

Oestrogen receptors 1042

Oestrogen replacement therapy 559

Oncofetal extracellular matrix 1071

Oncogene amplification 1328

Oral, gastric and colon cancer cells 416

Oral carcinoma 881, 1022

Oral contraceptives 918

Oral squamous cell carcinoma 1071

Orthotopic transplantation 934

Orthotopic tumours 1318

Ovarian cancer 152, 179, 631, 662, 733, 790, $855,1174,1304$

Ovarian neoplasms 559,654

Overall surival 1385

Overweight 890

P4 952

p16 677, 1122

p21 133

p21WAF1 959

p21wafl 252

p27Kip1 gene 1052

p53 179

p53 122, 133, 490, 702, 733, 959, 994, 1285

p53, canine 409

p53 mutants 212

Paclitaxel 416

Paediatric cancer 300

PAK-200S 1304

Pancreas-specific genes 350

Pancreatic cancer 80, 350

Papillomaviruses, human 554, 1234

PEG-m-THPC 631

Peptide 342

Perforin 359

Pericarditis 1037

Period effects 152

Peripheral blood 350

Peritonitis 1037
P-glycoprotein 783

P-glycoprotein inhibitors 942

pgp-170 416

Phagocytosis 37

Pharmacokinetic-pharmacodynamic

relationships 330

Pharmacokinetics 261, 1022, 1419

Phase I studies 1419

Phase I trials 760

Phase II studies 310

Phase II trials 457

Phenacetin 542

Phenotypic markers 952

Phosphofructokinase 219

Photodynamics 37

Photodynamic therapies $261,520,600,616$

Photosensitizers 261, 600

Phthalocyanines 616

Physiological factors 925

Phyto-oestrogens 1248

PK1-doxorubicin 99

Plasma ultrafiltrate 330

Plasminogens 1269

Platinum-based therapies 75

Pleural effusions 1059

Pleurisy 1037

PML 994

Pneumonitis 1037

Polymer conjugates 261

Polymorphism 179, 850

Population mixing 144

Population pharmacokinetics 99

Population-based screening 912

pPNETs-ETs 586

PR-39 393

Preclinical therapies 609

Preclinical toxicology 760

Predictive assays 354

Premenopause 167, 918

Prevention 1243

Primary chemotherapy 841

Prodrug therapy 24

Prognosis 122, 127, 133, 190, 469, 510, 662, $855,1052,1182,1213$

Prognostic factors 667, 747

Programme sensitivity 305

Progression 127

Prolactin-inducible proteins 1002

Proliferation 1017

Proliferative tumour cells 925

Proportionate incidences 912

Prostaglandin E 194

Prostate cancer 28, 242, 1052, 1269

Prostate cancer, androgen-dependent 28

Prostate cancer, androgen-independent 28

Prostate-specific antigens 490, 1269

Prostatic neoplasms 672, 1238

Proteases 790

Proteins 490

Protein kinase A 1134

Protein structures 1188

Proteolysis 287

Proteolytic cleavage 212

Proto-oncogenes 237

Proxy respondents 87

Purification 1188

$17 \mathrm{q} 241402$

Q-PCR-EIA 114

Quality of life 87 
Questionnaires 87

Quiescent tumour cells 925

RAD51 503

RAD52 503, 800

RAD54 503

Radiation 532

Radioimmunotherapy 972

Radiometabolic therapies 1378

Radiosensitivity 959

Radiotherapy 108, 323, 727, 841, 1196

Radon 900

Radon remediation 1243

Raf-1 1116

Randomization 1356

Ras 237

Ras 1116

Rat 631

RB phosphorylation 808

$\mathrm{Rb}$ protein 342,705

RBP-1 342

Rectal neoplasms 463

Registries 463

Relapse 727

Relapse-free survival 1385

Renal cancer 1009

Renal cell cancer 542, 741

Renal function 336

Residual diseases 860

Resistance 252

Response to chemotherapy 733

Retinoic acid 381

Retrospective cohort studies 144,1351

Retroviral transduction 43

Reverse transcription polymerase chain

reaction 350, 741, 870, 966, 1002, 1066

RT-PCR 350, 741, 870, 966, 1002, 1066

S phase 404

S phase fractions 1017

sCD30 1182

Schedules 1285

SCID mice 43, 622

Screening 305

Seascale 144

Seasonality 549

Second-line chemotherapy 846

Secondary myeloid malignancy 476

Selective COX-2 inhibitors 1274

Seroprevalence 893
Serosal complications 1037

SF2 354

s-ICAM-1 1182

Sigma-2 receptors 925

Single-chain antibodies 790

Skin 528

Small-cell lung cancer $667,1206,1213$

SN-38 1285

Sodium 28

Soft-tissue sarcomas 890,1017

Solid tumours 99

Soluble receptors 203

Somatostatin receptor 21402

South-east Asia 893

Southern blot analysis 1410

Soya 1248

Spinal cord tumours 835

Squamous cell carcinoma 95, 159, 510, 528

SSTR2 gene 1402

Stage III cancer 122

Staging 463

Standardization 870

Starting doses 760

Steroids 690

Steroid hormones 225,783

Stewart-Treves syndrome 532

Superantigens 359

Surgery $323,1206,1311$

Survival $323,528,727,850$

Survival analysis 463

Symptoms 1196

Syndecan 393

$t(14 ; 18) 860$

Targeting therapy 1155

T-cells 359

Testicular cancer incidence 1262

Thymidine phosphorylase 484

Thymomas 841

Tirapazamine 1127

Tissue inhibitor of matrix metalloproteinase (TIMP) 774

TNF- $\alpha 37$

Tobacco 1228

Tomudex 252

Topical application 13

Topoisomerase I 800

Topotecan 1304

Transcription 874

Transfection 647
Transitional cell carcinoma 638

Transplantation 476, 1344

Transurethral resection 832

Treatments 1351

Trivalent Fab' 972

Tumorigenesis 232

Tumorigenicity 756

Tumours 874

Tumours, human 75

Tumour antigens 19

Tumour cell growth 822

Tumour cell shedding 756

Tumour immunotherapy 431

Tumour inhibition 966

Tumour invasion 194, 381

Tumour markers 667,1059

Tumour perfusion 756

Tumour progression 242, 496

Tumour-rejection antigens 1080

Tumour responses 600

Tumour suppressor genes 1103, 1122, 1150

Tumour targeting 966, 972

Tumour therapies 359

Tumour vascular endothelium 1155

Tumour vasculature 261

Tumour-stromal interaction 194

Ultrasound imaging 520

United Kingdom 1243

uPAR 203

Urbanization 1262

Urinary bladder 1363

Vaccination 175

Vascular endothelial growth factor 54, 225 , $727,1335,1398$

VEGF 54, 225, 727, 1335, 1398

VEGF receptors 1335

VEGF-C 54

Weight gain 167

Western blotting 1080

wnt genes 496

Wortmannin 959

Xenografts 774

Yeast 800 